\title{
Theoretical investigation of amplified spontaneous emission in an active structure by extended $(3 \times 3)$ transfer matrix formalism: the case of a non-uniform longitudinal distribution of emitters
}

\author{
Yann G. Boucher \\ boucher@enib.fr
}

RESO Lab, ENIB, CS 73862, F-29238 Brest Cedex 3, France

We use an extended ( $3 \times 3$ ) Transfer Matrix Formalism (TMF) to investigate the properties of Amplified Spontaneous Emission (ASE) in an active structure that exhibits a non-uniform spatial distribution of emitters along its longitudinal axis. The spectral density of power of the internal and emitted fields, both filtered into the transfer function of the structure, are expressed analytically in structural dimensionless parameters. We show that a periodically modulated distribution of emitters inside a cavity, in spite of their being spatially non-coherent, are responsible for a cross-interference product that can lead to an unexpected resonant enhancement of the spectral selectivity. [D0I: $10.2971 /$ jeos.2006.06027]

Keywords: Amplified spontaneous emission, extended transfer matrix formalism, generalized transfer function, distributed feedback

\section{I n t rod uctio n}

For modelling one-dimensional (1D) active optoelectronic devices such as Semiconductor Optical Amplifiers (SOA) or laser oscillators, all-analytical formulas based on structural parameters are especially appealing. In this context, in 1991, Weber $\&$ Wang have suggested an original extended $(3 \times 3)$ Transfer Matrix Formalism (TMF) for dealing with internal sources in Distributed-Feedback (DFB) structures [1,2]. In the past few years, we have further developed this approach into the nonlinear regime, with special emphasis on gain saturation in SOAs [3] or threshold-crossing in single-mode lasers [4]. As a matter of fact, spontaneous emission is not only responsible for noise: it is also the very driving source enabling the laser oscillation to take place. Extended TMF enables one to derive easily the "Generalized Transfer Function" of the active resonator as introduced by Stéphan [5], that is, the spectral density of the (longitudinally averaged) internal field. Once integrated over its whole spectral range, the latter commands the saturation of the active medium: it becomes possible to derive self-consistently all-analytical expressions for the emitted power and linewidth, that remain continuously valid across threshold $[6,7]$.

In terms of modelling, the problem with active structures is that they add their intrinsic contribution to the signal fields. The relationship between input fields and output fields is no longer linear, in contrast with the usual $(2 \times 2)$ TMF [8]. The first advantage of extended $(3 \times 3)$ transfer matrices is that they retain their "cascadability", nonetheless. We work in the spectral domain in the frame of a semi-classical description of the electromagnetic field. Internal as well as emitted fields appear coupled into the spectral transfer function of the structure: even for an intrinsic "white" source, they are preferentially filtered into the resonance modes. This is not surprising: as pointed out by Kastler, the radiation emitted by active atoms placed inside a Fabry-Perot cavity is coupled into its Airy function [9]. The spatial and modal overlap between any emitter and the field is naturally taken into account by Extended TMF: enhancement or inhibition of its spontaneous emission with respect to its wavelength and position is derived as an obvious consequence.

As soon as we deal with an extended source, it is convenient to use the notion of "equivalent fields of spontaneous emission" that couple into the mode at both ends of each active zone [10]. These can be derived by longitudinal integration, in intensity, along the active zone, bearing in mind that the source is spatially incoherent. In the typical instance of uniformly distributed sources, only the amplitude of these two quantities has any experimental meaning, and their phase remain unspecified; since they are uncorrelated, their cross-product simply vanishes as soon as the spatial extension of the source is greater than about a half-wavelength. Nevertheless, in the general case of an arbitrary spatial distribution of emitters, this is not always true, as will appear below.

The chief result of the present paper consists of some previously unpublished calculations relative to active structures, with special emphasis on an arbitrary non-uniform longitudinal distribution of emitters. A limiting case will be especially considered: that of a periodic spatial modulation of the source in an otherwise homogeneous medium, placed inside a resonant cavity.

This paper is organised as follows: in Section 2, we briefly recall the principles of extended $(3 \times 3) \mathrm{TMF}$, and derive the expression of emitted and internal fields, as well as the general 
definition of equivalent fields. Section 3 is devoted to an arbitrary active zone placed inside an otherwise homogeneous cavity; under this simplifying assumption, all relevant quantities are formally derived, whatever the longitudinal distribution of emitters. In Section 4, we show that a spatially periodic distribution of emitters in an otherwise homogeneous cavity is responsible for a (possibly unheard-of) mechanism of spectral selectivity. Trends and conclusions are given in Section 5.

\section{EXTENDED TRANSFER MATRIX F O R M A L I S M}

Let us consider a one-dimensional active structure, as schematically depicted in Figure 1a. (a)

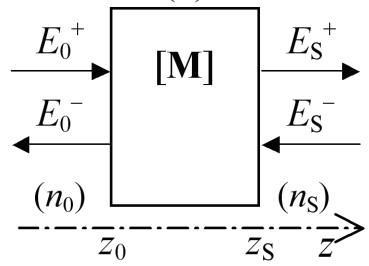

(b)

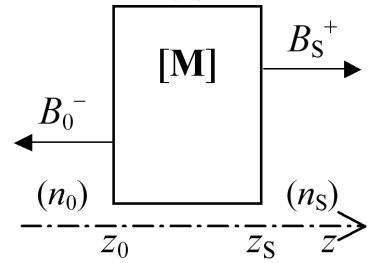

FIG. 1 Schematic depiction of a one-dimensional structure oriented along the z-axis, surrounded by two semi-infinite media of refractive (or effective) indices $\left(n_{0}, n_{S}\right)$.

(a) Transfer matrix $[\mathrm{M}]$ that connects the co- and contra-propagating components of the electric field $\left(E^{+}, E^{-}\right)$between abscissas $z_{0}$ and $z_{S}$ remains valid whatever the boundary conditions. (b) Even with no external input field $\left(E_{0}^{+}=E_{S}^{-}=0\right)$, "background" fields $\left(B_{0}^{-}, B_{S}^{+}\right)$are nevertheless emitted by the active structure.

If the structure is transversally single-mode, the field can be described as scalar for any given eigenstate of polarisation. The light propagates along the $z$-axis and the time dependence is $\exp (+i \omega t)$. Between abscissas $z_{0}$ and $z_{S}$, the co- and contrapropagating components of the electric part of the electromagnetic field are related by:

$$
\left(\begin{array}{c}
E_{0}^{+} \\
E_{0}^{-} \\
1
\end{array}\right)=\left(\begin{array}{ccc}
M_{11} & M_{12} & M_{13} \\
M_{21} & M_{22} & M_{23} \\
0 & 0 & 1
\end{array}\right)\left(\begin{array}{c}
E_{S}^{+} \\
E_{S}^{-} \\
1
\end{array}\right)
$$

where the first four coefficients $\left(M_{11}, M_{12}, M_{21}, M_{22}\right)$ are that of the usual $(2 \times 2)$ TMF in the notations of Yariv \& Yeh [8], whereas the third column contains the source terms. The transmission and reflection coefficients, in complex amplitude, for a wave coming from the left to the right, are respectively $t=1 / M_{11}$ and $r=\left(M_{21} / M_{11}\right)$. Similarly, the transmission and reflection coefficients from the right to the left are $t^{\prime}=\operatorname{det}(M) / M_{11}$ and $r^{\prime}=-M_{12} / M_{11}$. For the sake of clarity, let us assume that the two semi-infinite media surrounding the structure share the same refractive (or effective) index: $n_{0}=n_{S}$. In that case, $\operatorname{det}(M)=1$, and the transmission/reflection coefficients in intensity are $R=|r|^{2}, R^{\prime}=$ $\left|r^{\prime}\right|^{2}, T=T^{\prime}=|t|^{2}=1 /\left|M_{11}\right|^{2}$.

Even with no input wave $\left(E_{0}^{+}=E_{S}^{-}=0\right)$, "background" output waves $\left(B_{S}^{+}, B_{0}^{-}\right)$are emitted [Figure $\left.1 b\right]$. By applying the proper boundary conditions, we get:

$$
\left(\begin{array}{c}
0 \\
B_{0}^{-} \\
1
\end{array}\right)=\left(\begin{array}{ccc}
M_{11} & M_{12} & M_{13} \\
M_{21} & M_{22} & M_{23} \\
0 & 0 & 1
\end{array}\right)\left(\begin{array}{c}
B_{S}^{+} \\
0 \\
1
\end{array}\right) .
$$

A straightforward calculation leads to:

$$
\begin{gathered}
B_{S}^{+}=-M_{13} / M_{11}, \\
B_{0}^{-}=\left(-M_{13} M_{21}+M_{23} M_{11}\right) / M_{11} .
\end{gathered}
$$

The corresponding "background" spectral intensities, in $[W$. $\left.m^{-2} /\left(\mathrm{rad} \cdot \mathrm{s}^{-1}\right)\right]$, are:

$$
\begin{gathered}
I_{B S}^{+}=\left(\epsilon_{0} / 2\right) c n_{S}\left\langle\left|B_{S}^{+}\right|^{2}\right\rangle, \\
I_{B 0}^{-}=\left(\epsilon_{0} / 2\right) c n_{0}\left\langle\left|B_{0}^{-}\right|^{2}\right\rangle .
\end{gathered}
$$

where the brackets stand for a time average over the specific integration time of the detector under consideration. It should be noted that both emitted fields are projected into the transfer function $T(\omega)=1 /\left|M_{11}\right|^{2}$ : whatever the intrinsic spectral density of power of an emitter, the output fields are affected by the spectral signature of the structure itself.

Once the source-terms $M_{i 3}$ are known, the active properties of the structure are completely settled. What remains to be seen is the way to determine them. Let us consider first a single emitter localised at abscissa $z_{P}$ [Figure 2a].
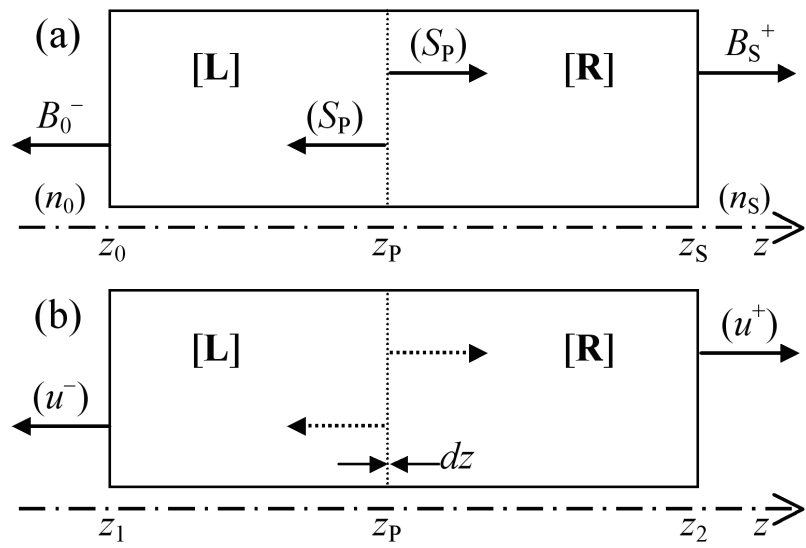

FIG. 2 Active structure. (a) Localised emitter at abscissa $z_{P}$; $[\mathrm{L}]$ and $[\mathrm{R}]$ denote the partial transfer matrices, from $z_{0}$ to $z_{P}^{-}$and from $z_{P}^{+}$to $z_{S}$, respectively. (b) Continuously extended source, between abscissa $z_{1}$ and $z_{2}$ : equivalent fields $\left(u^{+}\right)$and $\left(u^{-}\right)$that couple into the mode at both ends can be obtained by longitudinal integration over the whole active zone.

The transfer matrix $[\mathbf{M}]$ reads:

$$
[M]=\left(\begin{array}{ccc}
L_{11} & L_{12} & 0 \\
L_{21} & L_{22} & 0 \\
0 & 0 & 1
\end{array}\right)\left(\begin{array}{ccc}
1 & 0 & -\left(S_{P}\right) \\
0 & 1 & +\left(S_{P}\right) \\
0 & 0 & 1
\end{array}\right)\left(\begin{array}{ccc}
R_{11} & R_{12} & 0 \\
R_{21} & R_{22} & 0 \\
0 & 0 & 1
\end{array}\right),
$$

where $[\mathbf{L}]$ and $[\mathbf{R}]$ denote the "left" and "right" partial matrices, from $z_{0}$ to $z_{p}^{-}$and from $z_{P}^{+}$to $z_{S}$, respectively. We assume implicitly that all other effects of the emitter upon the incoming waves (such as dephasing, amplifying or scattering) can be safely neglected. Were it not the case, the central matrix in Eq.(7) would only have to be modified accordingly, without 
changing anything else. We'll keep the present form for the sake of simplicity. The source terms are therefore:

$$
\begin{aligned}
& M_{13}=-\left(S_{P}\right)\left(L_{11}-L_{12}\right), \\
& M_{23}=-\left(S_{P}\right)\left(L_{21}-L_{22}\right),
\end{aligned}
$$

so that, for instance, the emitted field and intensity at $z=z_{S}$ are:

$$
\begin{gathered}
B_{S}^{+}=\left(S_{P}\right)\left(L_{11}-L_{12}\right) / M_{11}, \\
I_{B S}^{+}=I_{P} T(\omega)\left|L_{11}-L_{12}\right|^{2},
\end{gathered}
$$

with $I_{P}=\left(\epsilon_{0} / 2\right) c n\left\langle\left|\left(S_{P}\right)\right|^{2}\right\rangle$ the "intrinsic" spectral intensity of the emitter. The last quantities are best expressed in terms of structural parameters:

$$
\begin{gathered}
B_{S}^{+}=\left(S_{P}\right) \frac{t_{R}\left(1+r_{L}^{\prime}\right)}{1-r_{L}^{\prime} r_{R}}, \\
I_{B S}^{+}=I_{P}\left|\frac{t_{R}\left(1+r_{L}^{\prime}\right)}{1-r_{L}^{\prime} r_{R}}\right|^{2},
\end{gathered}
$$

with $t_{R}=1 / R_{11}$ (resp. $r_{R}=R_{21} / R_{11}$ ) the complex transmittance (resp. reflectance) of the right block [R], and $r_{L}^{\prime}=$ $-L_{12} / L_{11}$ the complex reflectance of the left block [L], both taken as seen by the emitter.

The emitted field and intensity at $z=z_{0}$ are symmetrically given as:

$$
\begin{gathered}
B_{0}^{-}=\left(S_{P}\right) \frac{t_{L}^{\prime}\left(1+r_{R}\right)}{1-r_{L}^{\prime} r_{R}}, \\
I_{B 0}^{-}=I_{P}\left|\frac{t_{L}^{\prime}\left(1+r_{R}\right)}{1-r_{L}^{\prime} r_{R}}\right|^{2},
\end{gathered}
$$

with $t_{L}^{\prime}=\operatorname{det}(L) / L_{11}$ the complex transmittance, from the right to the left, of the left block [L]. We would like to emphasise that the left-wise and right-wise source contributions are coherent: the probability of spontaneous emission to the right and to the left can interfere mutually [11]; in other words, we are dealing with probability amplitude transfer matrices [12]. This effect can be especially important if the emitter is localised near a reflector, or inside a highly resonant microcavity.

Let us consider now an extended active zone $[\mathbf{Z}]$ of length $L$, located between abscissas $z_{1}$ and $z_{2}=z_{1}+L$ [Figure $2 b$ ]. Its overall contribution to the field emitted at both ends can be obtained by (incoherent) longitudinal integration, in intensity, over all emitters, with the formal replacement: $\left|\left(S_{P}\right)\right|^{2} \rightarrow$ $|A(z)|^{2} d z$.

The result depends on the lineic and spectral density $D(\omega, z)$ of the intensity of spontaneous emission, expressed in $[W$. $\left.m^{-2} \cdot m^{-1} /\left(\mathrm{rad} \cdot \mathrm{s}^{-1}\right)\right]$ :

$$
D(\omega, z)=\left(\epsilon_{0} / 2\right) c n\left\langle|A(z)|^{2}\right\rangle=\beta_{s p} \hbar \omega r_{s p}(\omega)
$$

where $\beta_{s p}$ denotes the coupling factor of spontaneous emission into the mode, $\hbar \omega$ the photon energy [J],n the refractive index of the active zone and $r_{s p}(\omega)$ its spectral rate of spontaneous emission $\left[\mathrm{s}^{-1} \cdot \mathrm{m}^{-3} /\left(\mathrm{rad} \cdot \mathrm{s}^{-1}\right)\right]$. Equivalent fields $\left(u^{+}\right)$ and $\left(u^{-}\right)$couple into the mode at both ends of the active zone.
With unspecified phase, they are only determined through average quadratic properties:

$$
\left\langle\left|\left(u^{+}\right)\right|^{2}\right\rangle=\left|1 / Z_{11}\right|^{2} \int d z\left\langle|A(z)|^{2}\right\rangle\left|\left(L_{11}-L_{12}\right)\right|^{2},
$$

$$
\left\langle\left|\left(u^{-}\right)\right|^{2}\right\rangle=\left|1 / Z_{11}\right|^{2} \int d z\left\langle|A(z)|^{2}\right\rangle|\operatorname{det}(L)|^{2}\left|\left(R_{11}-R_{12}\right)\right|^{2},
$$

$$
\begin{gathered}
\left\langle\left(u^{+}\right)\left(u^{-}\right)^{*}\right\rangle=\left|1 / Z_{11}\right|^{2} \int d z\left\langle|A(z)|^{2}\right\rangle\left(L_{11}-L_{12}\right) . \\
\operatorname{det}(L)^{*}\left(R_{11}-R_{12}\right)^{*}
\end{gathered}
$$

An elementary matrix manipulation enables one to express the source terms

$$
\begin{gathered}
Z_{13}=-\left(u^{+}\right) Z_{11}, \\
Z_{23}=-\left(u^{+}\right) Z_{21}+\left(u^{-}\right)
\end{gathered}
$$

These expressions hold whatever the precise form of $D(\omega, z)$ or the details of the structure. Let us concentrate now on an active zone inserted between two passive reflectors.

\section{EXTENDED ACTIVE ZONE IN- SIDE A C A VITY}

Two passive reflectors, respectively described by transfer matrices $[\mathbf{A}]$ and $[\mathbf{B}]$, surround the active zone $[\mathbf{Z}]$, as schematically depicted in Figure 3a.
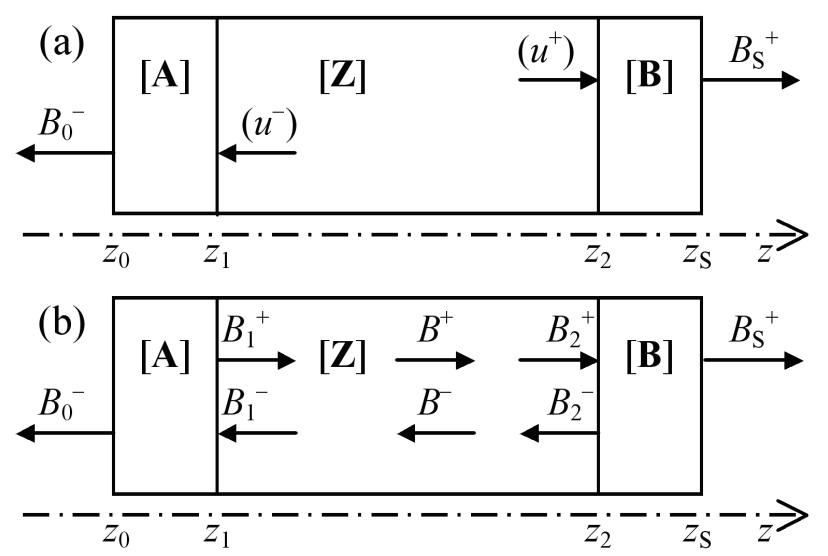

FIG. 3 Schematic depiction of an extended active zone placed inside a microcavity, delimited by two passive (source-less) reflectors $[\mathbf{A}]$ and [B]. (a) Equivalent fields $\left(u^{+}\right)$, $\left(u^{-}\right)$couple into the mode at both ends of the active zone after a single pass. (b) The actual value of the internal boundary fields at abscissas $z_{1}^{+}$and $z_{2}^{-}$, actually affected by multiple round-trips, depends upon, but should not be confused with $\left(u^{+}\right)$and $\left(u^{-}\right)$. Internal fields $\left(B^{+}, B^{-}\right)$can be expressed in terms of boundary fields $\left(B_{1}^{+}, B_{2}^{-}\right)$.

We limit ourselves to the ideal case where the active zone in itself is assumed totally devoid of any scattering centre, or small-scale index variation, that would be responsible for a coupling between co- and contra-propagating waves. As a consequence, its usual source-less $(2 \times 2)$ transfer matrix is 
purely diagonal, and the total extended $(3 \times 3)$ transfer matrix reads:

$[\mathrm{M}]=\left(\begin{array}{ccc}A_{11} & A_{12} 0 \\ A_{21} & A_{22} 0 \\ 0 & 0 & 1\end{array}\right)\left(\begin{array}{ccc}e^{+i \beta L} & 0 & -\left(u^{+}\right) e^{+i \beta L} \\ 0 & e^{-i \beta L} & +\left(u^{-}\right) \\ 0 & 0 & 1\end{array}\right)\left(\begin{array}{ccc}B_{11} B_{12} 0 \\ B_{21} B_{22} 0 \\ 0 & 0 & 1\end{array}\right)$

with $Z_{22}=1 / Z_{11}=t_{Z} \equiv \exp (-i \beta L)$, complex transmittance of the active zone.

Some obvious matrix algebra leads eventually to the expression of both emitted fields:

$$
\begin{aligned}
& B_{S}^{+}=t_{B}\left\{\frac{\left(u^{+}\right)+\left(u^{-}\right) r_{A}^{\prime} e^{-i \beta L}}{1-r_{A}^{\prime} r_{B} e^{-2 i \beta L}}\right\}, \\
& B_{0}^{-}=t_{A}^{\prime}\left\{\frac{\left(u^{-}\right)+\left(u^{+}\right) r_{B} e^{-i \beta L}}{1-r_{A}^{\prime} r_{B} e^{-2 i \beta L}}\right\},
\end{aligned}
$$

with $t_{A}^{\prime}=\operatorname{det}(A) / A_{11}, r_{A}^{\prime}=-A_{12} / A_{11}, t_{B}=1 / B_{11}, r_{B}=$ $B_{21} / B_{11}$ according to the usual notations. Note that the denominator is the obvious signature of a cavity resonance. With $t_{A}=1 / A_{11}$, the transfer function (or complex transmittance) of the structure reads:

$$
t=\frac{1}{M_{11}}=\frac{t_{A} t_{B} e^{-i \beta L}}{1-r_{A}^{\prime} r_{B} e^{-2 i \beta L}} .
$$

Internal fields at any abscissa $z \in\left[z_{1}, z_{2}\right]$ are easily expressed with respect to boundary fields $\left(B_{1}^{+}, B_{2}^{-}\right)$as drawn in Figure $3 b$ :

$$
\begin{gathered}
B^{+}(z)=B_{1}^{+} \exp \left[-i \beta\left(z-z_{1}\right)\right], \\
\left.B^{-}(z)=B_{2}^{-} \exp \left[-i \beta z-z_{2}\right)\right],
\end{gathered}
$$

with

$$
\begin{aligned}
& B_{1}^{+}=r_{A}^{\prime}\left\{\frac{\left(u^{-}\right)+\left(u^{+}\right) r_{B} e^{-i \beta L}}{1-r_{A}^{\prime} r_{B} e^{-2 i \beta L}}\right\}, \\
& B_{2}^{-}=r_{B}\left\{\frac{\left(u^{+}\right)+\left(u^{-}\right) r_{A}^{\prime} e^{-i \beta L}}{1-r_{A}^{\prime} r_{B} e^{-2 i \beta L}}\right\},
\end{aligned}
$$

Note that equivalent fields $\left\{\left(u^{+}\right),\left(u^{-}\right)\right\}$should never be confused with the actual values of $\left\{B_{2}^{+}, B_{1}^{-}\right\}$that result from multiple round-trips inside the cavity - except for the obvious case where there is no cavity $\left(r_{A}=r_{A}^{\prime}=r_{B}=r_{B}^{\prime}=0, t_{A}=\right.$ $\left.t_{A}^{\prime}=t_{B}=t_{B}^{\prime}=1\right)$.

Note also that the single emitter localised at abscissa $z_{P}=$ $z_{1}+d$ can be thought of as a particular instance of the most general case, with $D(\omega, z)=I_{P}(\omega) \delta\left(z-z_{P}\right)$. Its "equivalent fields" are straightforward:

$$
\begin{gathered}
\left(u^{+}\right)=\left(S_{P}\right) \exp [-i \beta(L-d)], \\
\left(u^{-}\right)=\left(S_{P}\right) \exp (-i \beta d),
\end{gathered}
$$

so that:

$$
\begin{gathered}
B_{S}^{+}=t_{B}\left(S_{P}\right) e^{-i \beta L} e^{+i \beta d}\left\{\frac{1+r_{A}^{\prime} e^{-2 i \beta d}}{1-r_{A}^{\prime} r_{B} e^{-2 i \beta L}}\right\}, \\
B_{0}^{-}=t_{A}^{\prime}\left(S_{P}\right) e^{-i \beta L} e^{+i \beta(L-d)}\left\{\frac{1+r_{B} e^{-2 i \beta(L-d)}}{1-r_{A}^{\prime} r_{B} e^{-2 i \beta L}}\right\} .
\end{gathered}
$$

The internal field $B_{\text {total }}(z)=B^{+}(z)+B^{-}(z)$ exhibits the characteristic behaviour of a standing wave. It is not difficult to check that for the maximum intensity to be emitted, two conditions should be simultaneously verified: (i) the wavelength should correspond to a resonance mode of the cavity (minimal value of the denominator); (ii) the single emitter should be located on an antinode of the standing wave (maximal value of the longitudinal overlap factor).

Formally, internal as well as emitted intensities can always be expressed as a combination of three basic components:

$$
\begin{gathered}
I_{U(+)}=\left(\epsilon_{0} / 2\right) c n\left\langle\left|\left(u^{+}\right)\right|^{2}\right\rangle, \\
I_{U(-)}=\left(\epsilon_{0} / 2\right) c n\left\langle\left|\left(u^{-}\right)\right|^{2}\right\rangle, \\
I_{U(+/-)}=\epsilon c n \operatorname{Re}\left[\left\langle\left(u^{+}\right)\left(u^{-}\right) *\right\rangle\right] .
\end{gathered}
$$

The first two terms are easily interpreted as the intrinsic intensities of Amplified Spontaneous Emission that would flow out of the active zone to the right and to the left, if both ends were perfectly adapted. The third term is a cross-interference term between fields of ASE emitted in opposite directions; it can only be observed if a resonator is involved.In order to determine these three quantities, we have only to evaluate the integral expressions of Eqs.(17)-(19) from $z_{1}=0$ to $z_{2}=L$, with $L_{11}=\exp (i \beta z)=1 / L_{22}, R_{11}=\exp [i \beta(L-z)]=1 / R_{22}$, $L_{12}=L_{21}=0$ and $R_{12}=R_{21}=0$. Since we deal with complex numbers, we should not forget that the modal propagation constant $\beta$ is also a complex number: let us write $\beta=\beta^{\prime}+i(g / 2)$, where $\beta^{\prime}=n \omega / c=2 \pi \lambda$ is the real wavevector and $g=\left(1 / I^{+}\right)\left(\partial I^{+} / \partial z\right)=\left(-1 / I^{-}\right)\left(\partial I^{-} / \partial z\right)$ is the modal gain $\left[\mathrm{m}^{-1}\right]$. The formal replacement leads to:

$$
\begin{gathered}
\left\langle\left|\left(u^{+}\right)\right|^{2}\right\rangle=\int_{z \in[0, L]} d z\left\langle|A(z)|^{2}\right\rangle e^{g(L-z),} \\
\left\langle\left|\left(u^{-}\right)\right|^{2}\right\rangle=\int_{z \in[0, L]} d z\left\langle|A(z)|^{2}\right\rangle e^{g z}, \\
\left\langle\left(u^{-}\right)\left(u^{-}\right) *\right\rangle=e^{-i \beta L} \int_{z \in[0, L]} d z\left\langle|A(z)|^{2}\right\rangle e^{+2 i \beta^{\prime} z} .
\end{gathered}
$$

With respect to $D(z)$, the three intensities are:

$$
\begin{gathered}
I_{U(+)}=\int_{z \in[0, L]} d z D(z) e^{g(L-z)}, \\
I_{U(-)}=\int_{z \in[0, L] d z} D(z) e^{g z}, \\
I_{U(+/-)}=2 \operatorname{Re}\left[e^{-i \beta L} \int_{z \in[0, L]} d z D(z) e^{+2 i \beta^{\prime} z}\right] .
\end{gathered}
$$

The first two terms can be formally expressed, if necessary, in terms of Laplace Transforms of the spatial source distribution, whereas the third term is more obviously related to its Fourier Transform. $D(z)$ is a real-valued function of $z$ defined on $[0, L]$ that can always be written as:

$$
D(z)=\int_{R} d \mu D(\mu) e^{-2 i \pi \mu z},
$$

with a spatial spectrum $D(\mu)$ inversely expressed as:

$$
D(\mu)=\int_{R} d z D(z) e^{+2 i \pi \mu z},
$$

so that

$$
I_{U(+/-)}=2 \operatorname{Re}\left[e^{-i \beta L} D\left(\beta^{\prime} / \pi\right)\right] .
$$

We can see that the frequency-dependent cross-interference term $I_{U(+/-)}$, which represents a spectral density of intensity, 
is directly related to the spatial Fourier Transform of the longitudinal distribution of emitters.

For instance, if the active zone is uniform, $D(z)=$ $\beta_{s p} \hbar \omega r_{s p}(\omega)=D_{0}$ constant over $[0, L]$, and $D(z)=0$ outside this interval. It is easy to see that $D\left(\beta^{\prime} / \pi\right)=$ $D_{0} e^{2 i \pi L / \lambda} \operatorname{Linc}(2 \pi L / \lambda)$, where the sine-cardinal is defined by $\operatorname{sinc}(0)=1$ and $\operatorname{sinc}(x)=\sin (x) / x$ elsewhere. As soon as $L$ is greater than about $\lambda / 4$, the cross-interference term is rapidly washed out to zero. In most amplifier or laser systems, the bulk active zone is much greater than the wavelength, and $I_{U(+/-)}$ simply vanishes. On the other hand, in a VerticalCavity Surface-Emitting Laser (VCSEL), the typical width of each Quantum Well (QW) in the active zone is only $10 \mathrm{~nm}$, much smaller than a wavelength: each emitter can be thought as strictly localised.

Since the case of uniform active zones remains very important in practice, we feel useful to give an explicit expression for the longitudinally averaged internal intensity $I_{A V}(\omega)$ with respect to $I_{U}(\omega)=I_{U(+)}=I_{U(-)}$. Neglecting the standingwave term, $\left|B_{\text {total }}\right|^{2}$ is replaced by $\left|B^{+}\right|^{2}+\left|B^{-}\right|^{2}$, and we get eventually:

$$
\begin{aligned}
& I_{U}(\omega)=D_{0} L\left(\frac{e^{g L}-1}{g L}\right)=\beta_{s p} \hbar \omega r_{s p}(\omega) L\left(\frac{e^{g L}-1}{g L}\right), \\
& I_{A V}(\omega)=I_{U}(\omega)\left\{\frac{R_{A}^{\prime}+R_{B}+2 R_{A}^{\prime} R_{B} e^{g L}}{\left|1-r_{A}^{\prime} r_{B} e^{-2 i \beta L}\right|^{2}}\right\}\left(\frac{e^{g L}-1}{g L}\right),
\end{aligned}
$$

with $R_{A}^{\prime}=\left|r_{A}^{\prime}\right|^{2}, R_{B}=\left|r_{B}\right|^{2}$. In a symmetric structure $\left(R_{A}^{\prime}=R_{B}=R_{0}\right)$ this simplifies into:

$$
I_{A V}(\omega)=I_{U}(\omega)\left\{\frac{2 R_{0}\left(1+e^{g L}\right)}{\left|1-r_{A}^{\prime} r_{B} e^{-2 i \beta L}\right|^{2}}\right\}\left(\frac{e^{g L}-1}{g L}\right) .
$$

In a single-mode laser structure, the saturating intensity $I_{S A T}\left[\mathrm{~W} \cdot \mathrm{m}^{-2}\right]$ would be obtained by spectral integration of $I_{A V}(\omega)$ over its whole spectral range.

\section{RESONANT PERIODIC DISTRI- B U TION O F E MITTERS}

As a last example, let us consider the case of a periodically modulated distribution of sources. To be more specific, we assume a symmetric $\Lambda$-periodic distribution of $N$ identical localised emitters, in a manner somewhat reminiscent of the Resonant-Periodic Gain scheme to be found in some VCSELs $[13,14]$, except that here, the gain is supposed uniformly distributed along the active zone: only the emitters are discrete. The equivalent fields are:

$$
\begin{gathered}
\left(u^{+}\right)=e^{-i N \beta \Lambda} \sum_{q}\left(S_{q}\right) e^{i \beta \Lambda / 2} e^{i(q-1) \beta \Lambda}, \\
\left(u^{-}\right)=\sum_{p}\left(S_{p}\right) e^{-i \beta \Lambda / 2} e^{-i(p-1) \beta \Lambda},
\end{gathered}
$$

with $L=N \Lambda$ and $\forall(p, q) \in\{1,2, \ldots, N\}^{2},\left\langle\left(S_{p}\right)\left(S_{q}\right)^{*}\right\rangle=$ $\left|S_{0}\right|^{2} \delta_{p q}$, where $\delta_{p q}$ is the Kronecker symbol: since different emitters are not spatially coherent, they do not interfere with each other. They emit the same intensity $I_{0}=$ $\left(\epsilon_{0} / 2\right) c n\left\langle\left|\left(S_{p}\right)\right|^{2}\right\rangle$.
After some calculations, we get eventually:

$$
\begin{gathered}
\left\langle\left(u^{+}\right)\left(u^{-}\right)^{*}\right\rangle=\left|S_{0}\right|^{2} e^{g L / 2}\left\{\frac{\sin \beta^{\prime} L}{\sin \beta^{\prime} \Lambda}\right\}, \\
I_{U(+/-)}=I_{0} e^{g L / 2}\left\{\frac{\sin \beta^{\prime} L}{\sin \beta^{\prime} \Lambda}\right\} .
\end{gathered}
$$

The function $\sin (N x) / \sin (x)$ is drawn in Figure 4 .

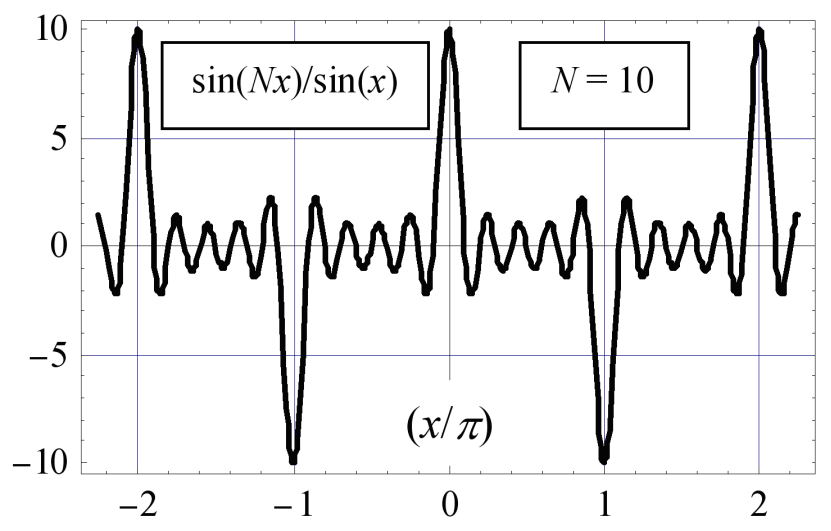

FIG. 4 Function $F(x)=\sin (N x) / \sin (x)$, with $N=10$. A pronounced enhancement is obtained on each resonance ( $x=m \pi$, with $m$ integer). Note that the crossinterference term can change sign, according to the parity of integer $m$.

The maximum $I_{U(+/-) M A X}=N I_{0} e^{g L / 2}$ of this crossinterference term is reached for wavelengths that are in resonance with the period: the resonance condition is $\Lambda=(2 m+$ $1)(\lambda / 2)$, with $m$ integer. The lowest-energy resonance is obtained for $\lambda=2 \Lambda$, which corresponds to the traditional firstorder Bragg wavelength, as defined in Distributed Feedback structures with respect to the period $\Lambda$ [15]. In contrast with a uniform distribution, this effect is all the more pronounced as the number of periods, hence the overall length, is greater. We would like to emphasise, however, that this specific spectral enhancement has nothing to do with either index-coupling or gain-coupling, since both index and gain are assumed completely uniform: only the source distribution is considered periodic (whether that restrictive assumption is realistic or not is another matter). We wouldn't call that mechanism "sourcecoupling", however tempting the neologism, since it is quite different from the distributed feedback that couples waves propagating in both directions. What we are considering is a pure interference effect. On the other hand, "Resonant Periodic Distribution of Emitters", or "Resonant Periodic Source" for short, seems a correct designation.

Note also that $I_{U(+/-)}$ is not necessarily positive: indeed, it can change sign according to the parity of the integer $m$. Its contribution is algebraic and the total emitted field also depends on the phase of the reflectors. Besides, the calculation was presented in a symmetric configuration, but we would like to point out that any translation of the emitters along the cavity axis would shift the phase of equivalent fields $\left(u^{+}\right)$and $\left(u^{-}\right)$, thus slightly modifying the balance between the various contributions.

Now why does a spatially incoherent source distribution $D(z)$ lead to a possibly non-vanishing cross-interference term? It 
should be remembered that the spontaneous contributions to the right and to the left of each emitter remain mutually coherent. The best analogy would be with a classical twopinholes Young interferometer: if we use an extended uniform source (instead of a point source), the fringe visibility decreases rapidly by increasing the source dimension. On the other hand, if we were to use a periodic series of pointsources, all giving rise to the same interference pattern, but translated exactly from an integer number of fringes, then we would continue to obtain brightly contrasted fringes - even if the total extension of the source becomes much greater than its coherence length.

\section{CONCLUSION AND PERSPEC- TIVES}

Extended $(3 \times 3)$ Transfer Matrix Formalism constitutes an elegant as well as powerful tool for modelling Amplified Spontaneous Emission in one-dimensional active structures. Each active zone is completely determined by its $(3 \times 3)$ transfer matrix, with two source terms $M_{i 3}$ that can always be expressed in terms of equivalent ("single-pass") spontaneous fields $\left\{\left(u^{+}\right),\left(u^{-}\right)\right\}$that couple into the mode at both ends of the active zone. An ordinary matrix product gives the overall transfer matrix. Emitted as well as internal fields appear projected into the spectral transfer function of the structure. Since the source is spatially incoherent, contributions from different emitters or active zones add in intensity. On the other hand, the equivalent spontaneous fields emitted to the right and to the left by the same emitter remain coherent and can therefore lead to observable interference. In an extended active zone, they can be responsible for a non-vanishing crossproduct $\left\langle\left(u^{-}\right)\left(u^{-}\right)^{*}\right\rangle$. In an otherwise homogeneous active zone, the spectral behaviour of the latter appears strongly related to the spatial Fourier Transform of the longitudinal distribution of emitters.

We have thoroughly described the limiting case of an idealised extended source totally devoid of backscattering, where only the emitters are (fictively) modulated; nevertheless, Eqs.(1) to (21) remain valid without restriction whatever the structure. The general case of a non-uniform distribution of emitters is but rarely considered, but we should mention that for DFB lasers, our results are consistent with an alternative model derived by Makino for ASE in gain-coupled QuantumWell DFB lasers, where the modulation of the source is explicitly taken into account in the frame of the Green function formalism [16].

Note also that all relevant quantities can be expressed either in terms of somewhat abstract matrix elements $M_{i j}$, or more palpably in terms of structural properties (reflection or transmission coefficients, position, and so on). No need to calculate separately the spectral or longitudinal overlap factor between the fields and the active zone, since they are naturally taken into account by the formalism.

Although we have restrained our study to the linear case, it should be pointed out that our all-analytical formulas for the averaged internal spectral density of intensity can easily be used in order to derive the (spectrally integrated) saturating intensity. Steady-state properties of the active structure can be investigated in the frame of Extended TMF well into the nonlinear regime, from gain saturation to single-mode laser oscillation.

Besides, it should be mentioned that the control of spontaneous emission in Photonic Crystals has always been an important issue $[17,18]$ : in one-dimensional periodic structures at least, we have good reason to believe that all-analytical expressions derived from ETMF could prove especially attractive for designing and tailoring almost-periodic active photonic devices.

\section{ACKNOW LEDGEMENTS}

The author would like to thank Pr. Le Bihan, Head of RESO Laboratory at École Nationale d'Ingénieurs de Brest, for his support and encouragement.

\section{References}

[1] J. P. Weber and S. Wang, IEEE J. Quantum Electron. 27 (10), 22562266 (1991).

[2] J. P. Weber, IEEE J. Quantum Electron. 29 (1), 296 (1993).

[3] Y. Boucher and A. Sharaiha, IEEE J. Quantum Electron. 36 (6), 708720 (2000).

[4] Y. Boucher, Recent Res. Devel. Optics 3, 177-204 (2003).

[5] G. M. Stéphan, Phys. Rev. A 55 (2), 1371-1384 (1997).

[6] G. M. Stéphan, Quantum Semiclass. Opt. 10, 1-12 (1998).

[7] Y. G. Boucher, S. Blin, P. Besnard, and G.M. Stéphan, Proc. SPIE, 5452, 654-665 (2004).

[8] A. Yariv and P. Yeh, Optical Waves in Crystals (Wiley, New York, 1984).

[9] A. Kastler, Appl. Optics 1 (1), 17-24 (1962).

[10] H. K. Choi, K. L. Chen, S. Wang, IEEE J. Quantum Electron. QE-20 (4), 385-393 (1984).

[11] P. Stehle, Phys. Rev. A 2 (1), 102-106 (1970).

[12] G. B. Morrison and D. T. Cassidy, IEEE J. Quantum Electron. 36 (6), 633-640 (2000).

[13] M. Y. A. Raja, S. R. J. Brueck, M. Osinski, C. F. Schaus, I. G. McInerney, T. M. Brennan and B. E. Hammons, IEEE J. Quantum Electron. QE-25 (6), 1500-1512 (1989).

[14] S. W. Corzine, R. S. Geels, J. W. Scott, R. H. Yan and L. A. Coldren, IEEE J. Quantum Electron. QE-25 (6), 1513-1524 (1989).

[15] H. Kogelnik and C. V. Shank, J. Appl. Phys. 43 (5), 2327-2335 (1972).

[16] T. Makino, IEEE J. Quantum Electron. 33 (6), 1010-1017 (1997).

[17] J. D. Joannopoulos, J. N. Winn, R. D. Meade, Photonic Crystals: Molding the Flow of Light (Princeton University Press, 1995; 2nd edition 2006).

[18] J. M. Lourtioz, H. Benisty, V. Berger and J. M. Gérard, Les cristaux photoniques, ou la lumière en cage (Hermès, Paris, 2003, in French); English version: Photonic Crystals: Toward Nanoscale Photonic Devices (Springer-Verlag, Berlin, 2005). 